\title{
Sawflies (Hymenoptera: Symphyta) of Cerová vrchovina Upland (South Slovakia)
}

\author{
Attila Balázs ${ }^{1} \&$ AtTIla Haris ${ }^{2}$ \\ ${ }^{1}$ Department of Zoology, Fisheries, Hydrobiology and Apiculture Mendel University in Brno, \\ CZ-613 00 Brno, Zemedelska 1, Czech Republic, e-mail: balazsaeko@gmail.com
}

2H-1076 Budapest, Garay street 19 2/20, Hungary, e-mail: attilaharis@yahoo.com

\begin{abstract}
Balázs, A. \& Haris, A.: Sawflies (Hymenoptera: Symphyta) of Cerová vrchovina Upland (South Slovakia). Abstract: 189 specimens of 75 species of sawflies were collected at Cerová vrchovina Upland (South Slovakia). Pseudocephaleia praeteritorum (Semenov, 1934), Aprosthema austriacum (Konow, 1892) and Euura calcicola (Benson, 1948) are new records for the Slovak fauna. Rare species are: Pseudocephaleia praeteritorum (Semenov, 1934), Aprosthema austriacum (Konow, 1892), Euura calcicola (Benson, 1948) and Fenusella glaucopis (Konow, 1907).
\end{abstract}

Keywords: Hymenoptera, Symphyta, Cerová vrchovina Upland, Slovakia, new records

\section{Introduction}

Territory of Cerová vrchovina Upland takes 16771 ha in the south of Central Slovakia, in the border of the former Nógrád and Gömör counties (Fig. 1). The area belongs to the Inner Western Carpathians and to the Matricum subarea from geobotanic point of view (Bolfík et al. 1990). Cerová vrchovina Upland belongs to the Bukovinka, Fil'akovo and Lučenec formations with varied clays, sands, gravels, rhyodacite tuffs, claystones, sandstones and gray calcareous siltstones formed with alkali basalts and basanites (BIELY et al. 2002). The highest peak is Karancs ( $728 \mathrm{~m})$, the highest volcano is Medves (575 m) and the lowest altitude is at Janice water reservoir (167 m) (HoRváTH \& GAÁloví 2007). Karancs Mts. are covered by continuous forests, settlements occurs on the margins only, whilst the woodlands at the Medves Mts. are fragmented and villages are distributed evenly within the area. The most common vegetation associations in the studied area are Prunetum fruticosae, Alnetum glutinosae, Carpinion betuli, Querco robori-Carpinetum, Carici pilosae-Carpinetum, Mercuriali-Tilietum, Melittio-Fagetum, Quercetum petraea-cerris (Figs. 2, 3 and 4) (CSIKY 2004). This unit is poor in wetlands and even the major watercourses have a low water discharge. The area is divided by a watershed between the rivers Danube and Tisza. Cerová Upland is situated in warm zone which has continental characteristics; warm, extremely dry district with moderate winter and warm, moderately dry district with cold winter (HoRváTH \& GAÁLOvé 2007).

From the region, 24 species were published from Gemerček (Kisgömöri) (the most interesting species are: Athalia rufoscutellata Mocsáry, 1879 and Tenthredo vespiformis 
(Schrank, 1781)) and further 12 species from Petrovce (Gömörpéterfala) (the most interesting species are Hoplocampa pectoralis C. G. Thomson, 1871 and Dolerus ferrugatus Serville, 1823) in the monograph titled Sawflies of the Carpathian Basin, History and Current Research (ROLLER \& HARIS 2008).

\title{
Material and methods
}

The research of sawflies started in 2018 and continued in 2019. The first author with Jan Bezděk and Vladimír Hemala spent 18 days on field, 6 days in April, 8 days in May and 2 days in June 2019 and 2 days in 2018. Most of the specimens was captured with sweeping net and mounted afterwards; the rest of the specimens were caught by Malaise trap and conserved in $80 \%$ alcohol. The material is maintained at the first author personal collection, some rare species was deposited in Rippl-Rónai Museum, Kaposvár.

For identification, Zhelochovtsev's work on the sawflies of the European part of the former USSR (ZHELOCHOVTSEV, 1988) was consulted. We also used some recent revisions and works to make the identifications even more precise (ACHTERBERG \& AARTSEN 1986, Blank \& Ritzau 1998, Haris 2001, 2006, Koch 1988, Zombori 2016).

For the discussion of the distribution of sawflies, we consulted the book of Roller and Haris titled Sawflies of the Carpathian Basin, History and Current Research (RoLler \& HARIS 2008), the most recent European checklist of species (TAEGER et al. 2006) augmented by other faunistic records from the Carpathian Basin (RoLLER 1993, 1994, 1996, 1998, 1999a, b, c, d, e, 2000a, b, c, 2001, 2004, 2005, 2006a, b, 2010, Roller \& LuKÁšs 1999. Roller et al. 2006, Roller \& MaceK 2017, Roller \& OlŠOvsKÝ 2012, Haris 2009, 2010, 2011, 2012, 2018a,b, HARIS \& GYURKOVICS 2012 ).

The higher classification of sawflies applied in this work follows the Hymenoptera part of Fauna Europaea (ACHTERBERG 2013).

\section{List of sites}

\author{
Abbreviations: \\ AB - Attila Balázs, VH - Vladimír Hemala, JB - Jan Bezděk \\ Čamovce (Csomatelke): Bodoszó, around 48¹4'40.790"N, 1953'2.158"E - AB. \\ C̆amovce (Csomatelke): Isten-hegye, around 48¹4' 19.758"N 19॰53' 23.016"E - AB. \\ Čamovce (Csomatelke): László hegy, around 48¹5' 34.383"N 1952' 59.166"E - AB. \\ C̆amovce (Csomatelke): Rákos, between $48^{\circ} 13^{\prime} 54.585^{\prime \prime} \mathrm{N}, 19^{\circ} 52^{\prime} 36.976^{\prime \prime} \mathrm{E}$ and $48^{\circ} 13^{\prime} 54.675^{\prime \prime} \mathrm{N} 19^{\circ} 52^{\prime}$ \\ $39.082 " \mathrm{E}-\mathrm{AB}$ \\ Čamovce (Csomatelke): Szentfali, around 48¹5'4.852"N, 1951'55.398"E - AB. \\ Čamovce (Csomatelke): Vidosza, around 48 $13^{\prime} 50.565^{\prime \prime N} 19^{\circ} 53^{\prime} 26.521^{\prime \prime E}$ - AB. \\ Chrámec (Harmac): Farkas-völgy, 48¹6'17.529"N, 20¹0'39.640"E - AB. \\ Gemerské Dechtáre (Détér): Nagymál-Bérc, 24. 05. 2019, around 48¹4' 33.983"N 2001' 34.050"E - AB, VH. \\ Hajnáčka (Ajnácskő), Tilic Hill, 48¹2'41.683"N, 1956'40.393"E - AB. \\ Hajnáčka (Ajnácskő): Sás Bikk 21. 04. 2019, around 48¹3' 45.548"N 1958' 12.482"E - AB. \\ Jestice (Jeszte), Nagy-Somos, $48^{\circ} 12^{\prime} 47.603^{\prime \prime} \mathrm{N}, 20^{\circ} 2^{\prime} 49.250^{\prime \prime} \mathrm{E}-\mathrm{AB}, \mathrm{JB}, \mathrm{VH}$. \\ Jestice (Jeszte): Várhegy, around 48¹2'53.516"N, 204'4.808"E - JB, AB. \\ Petrovce (Gömörpéterfala): Fenek, 48¹1'54.319"N, 20²'52.882"E - AB. \\ Petrovce (Gömörpéterfala): Malaise trap, $48^{\circ} 11^{\prime} 52.568^{\prime \prime} \mathrm{N}, 20^{\circ} 2^{\prime} 57.767 " \mathrm{E}-\mathrm{AB}$. \\ Petrovce (Gömörpéterfala): Pap-berki, around 48¹0' 55.038"N 2000' 42.468"E - AB.
}


Stará Bašta (Óbást), Nagy-mocsaras, 48¹1'29.441"N, 1957'9.728"E - AB.

Šurice (Söreg), below the Pogány castle, 48¹3'21.338"N, 1955'3.747"E - AB.

Tachty (Tajti) valley of Gortva stream, around 48 $09^{\prime} 03.359^{\prime \prime} \mathrm{N} 19^{\circ} 55^{\prime} 36.626^{\prime \prime E}$ - JB.

Tachty (Tajti) valley of Meleg-hill, around 48 $9^{\prime} 0.627^{\prime \prime N}, 19^{\circ} 55^{\prime} 48.136^{\prime \prime} \mathrm{E}-\mathrm{AB}$.

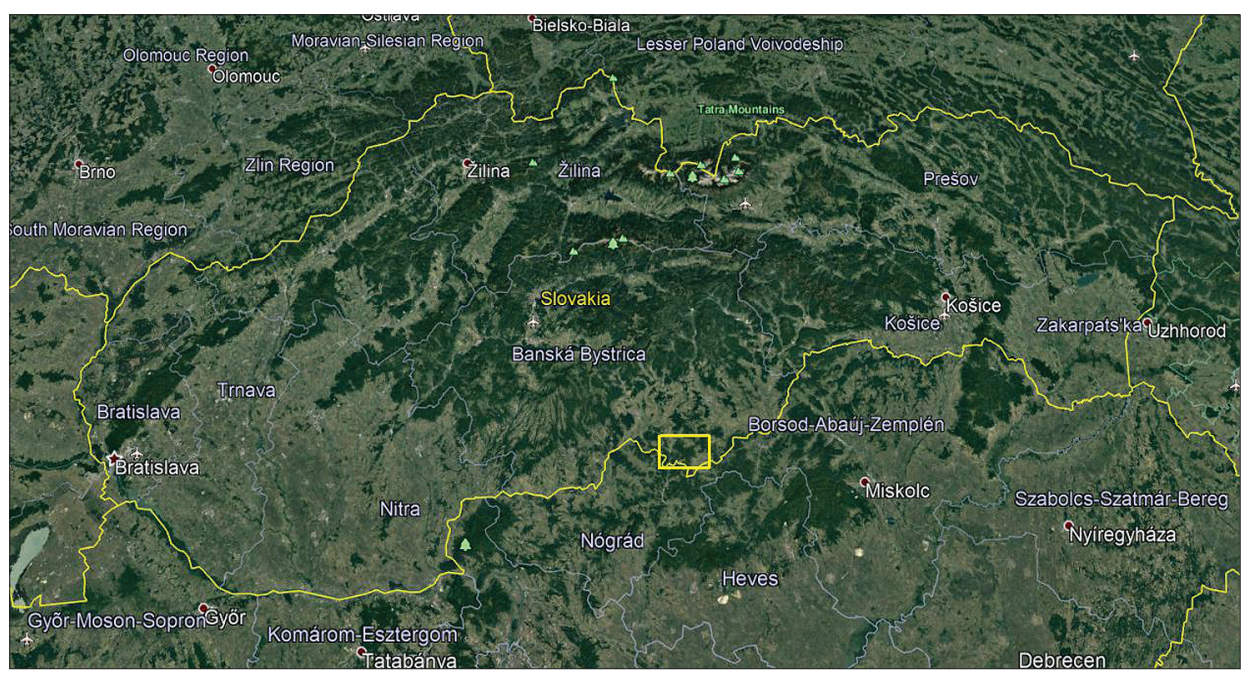

Fig. 1: Position of Cerová vrchovina Upland in Slovakia

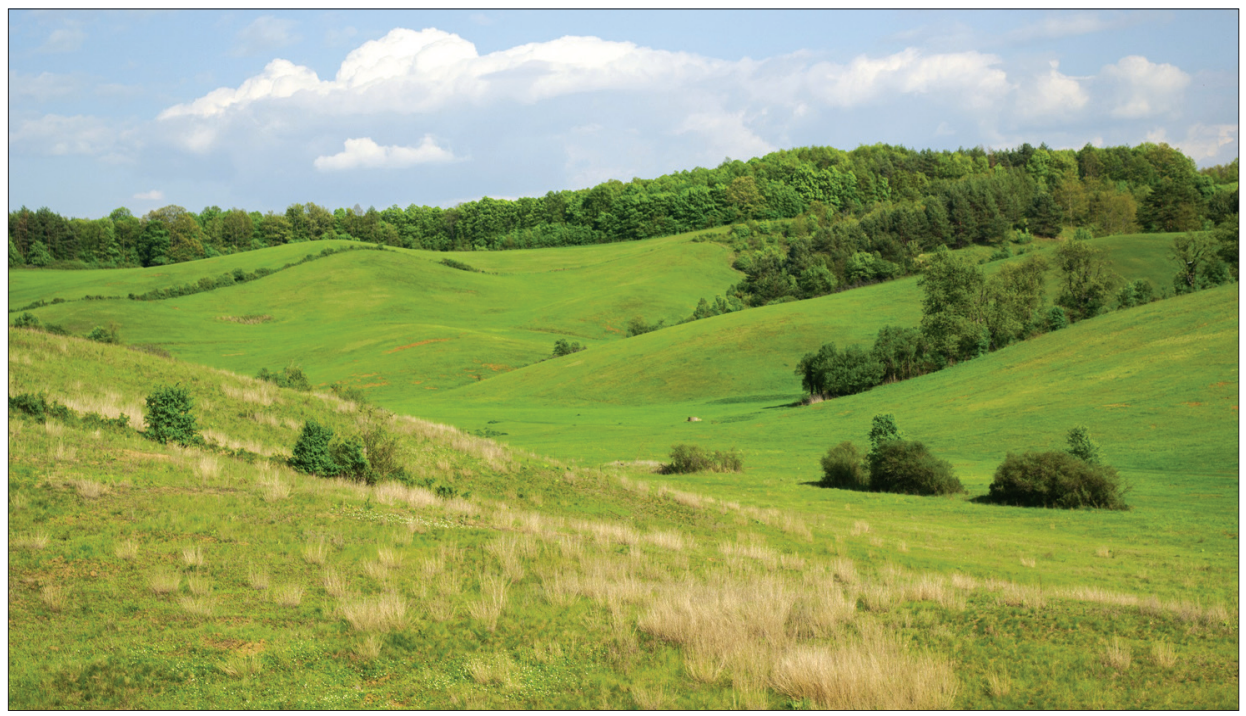

Fig. 2: Mesoxerophilous subcontinental meadowsteppes at Jestice (Jeszte), around Várhegy 


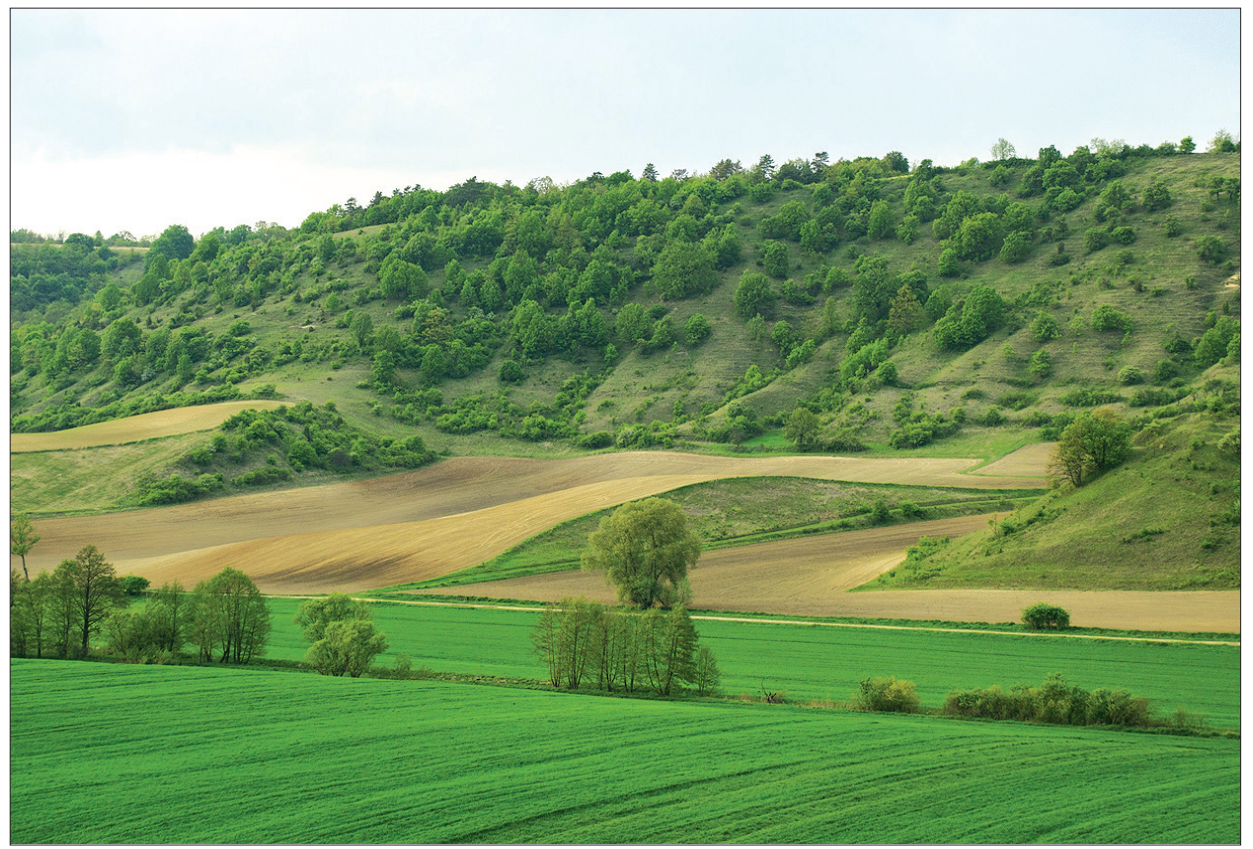

Fig. 3: Thermophilous scrub on sunny, stony slopes (Pruno spinosae-Crataegum) at Gemerské Dechtáre (Détér): Nagymál-Bérc

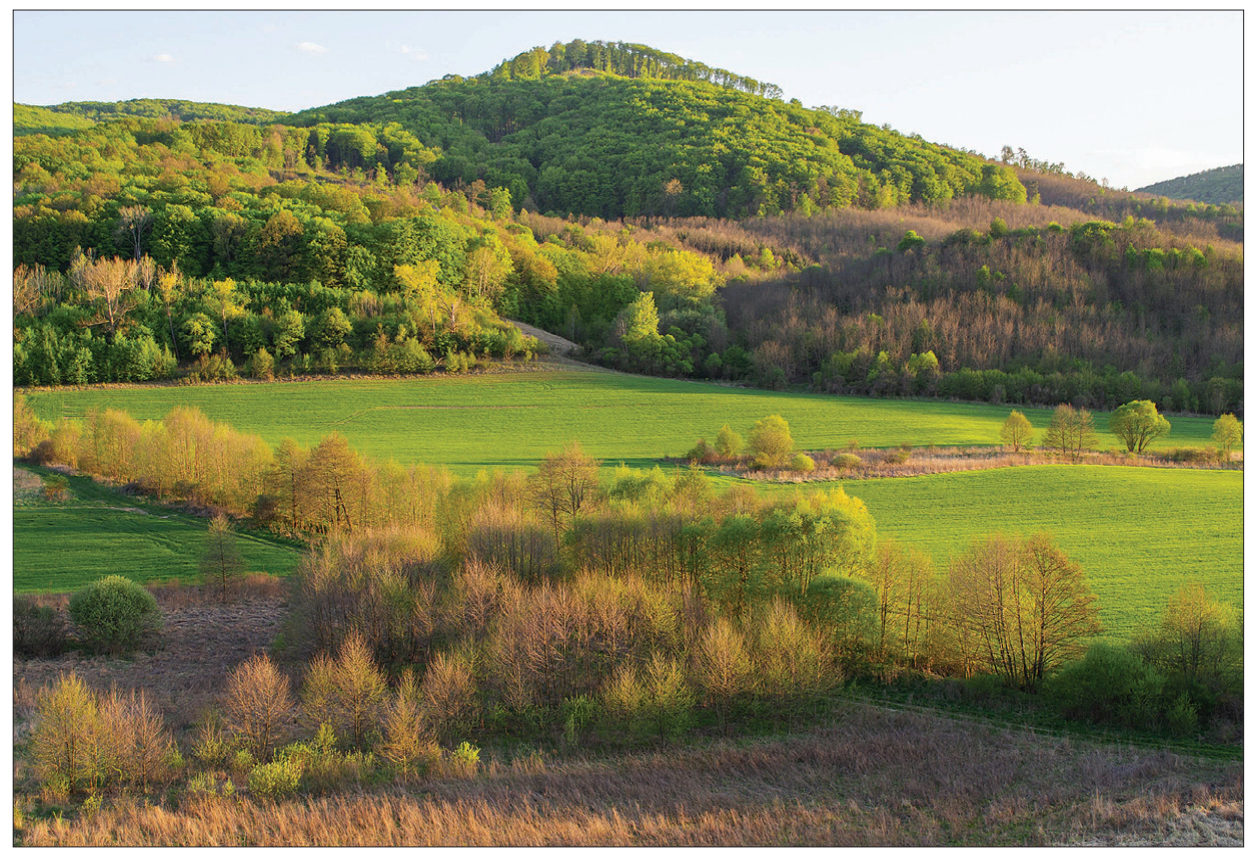

Fig. 4: Thermophilous woodlands with decidous oaks of eastern sub-Mediterranean regions, Šurice (Sőreg), view from Pohansky castle 


\section{Results}

\section{Xyelidae}

Xyela (Xyela) graeca J.P.E.F. Stein, 1876: Čamovce (Csomatelke): Rákos, 20. 04. 2019, 6 females, 5 males. Sporadic. Larva on Pinus nigra.

\section{Pamphiliidae}

Pseudocephaleia praeteritorum (Semenov, 1934): Jestice (Jeszte): Nagy-Somos 03. 05. 2019, female. Rare, new record for Slovakia. Hostplant unknown.

Neurotoma nemoralis (Linné, 1758): Šurice (Söreg): Bagoly-vár, 21. 04. 2019, 1 female. Known hostplants: Prunus mahaleb, P. armeniaca, P. spinosa and P. cerasus. Locally frequent pest.

\section{Orussidae}

Orussus abietinus (Scopoli, 1763): Hajnáčka (Ajnácskő): Sás Bikk 26. 05. 2019, 1 female. Sporadic. Parasitoid of Semanotus undatus L.

\section{Cephidae}

Calameuta (Calameuta) pallipes (Klug, 1803): Čamovce (Csomatelke): Bodoszó, 22. 05. 2019, 1 female; Jestice (Jeszte), 03. 05. 2019, 1 female; Čamovce (Csomatelke): László hegy, 24. 04. 2019, 2 females; Čamovce (Csomatelke): Bodoszó, 24. 05. 2019, 1 female. Frequent species. Hostplants: diverse Poaceae.

Cephus brachycercus Thomson, 1871: Hajnáčka (Ajnácskő): Sás Bikk 01. 05. 2019. 1 male. Widely distributed, sporadic species. Hostplant unknown.

Cephus pygmeus (Linné, 1767): Stará Bašta (Óbást), Nagy- mocsaras, 09. 06. 2019, 1 male; Šurice (Söreg), 21. 05. 2018, 1 male. Common. Insect pest of cereals and Gramineae.

Cephus spinipes (Panzer, 1800): Hajnáčka (Ajnácskő): Sás Bikk 26. 05. 2019, 2 males; Čamovce (Csomatelke): Bodoszó, 22. 05. 2019, 1 female. Frequent species. Known hostplant: Phleum pratense.

\section{Argidae}

Aprosthema austriacum (Konow, 1892): Čamovce (Csomatelke): Isten-hegye, 20. 04. 2019, 1 female. Rare. Hostplant unknown. New record for Slovakia.

Arge cyanocrocea (Förster, 1771): Šurice (Söreg): below the Pogány castle, 21. 05. 2018, 1 female. Common species. Known hostplants: Rubus idaeus and Sanguisorba officinalis. Arge melanochra (Gmelin, 1790): Stará Bašta (Óbást), Nagy-mocsaras 09. 06. 2019,

1 male. Frequent. Hostplant: Crataegus oxycantha.

Arge nigripes (Retzius, 1783): Hajnáčka (Ajnácskő): Sás Bikk 30. 04. 2019, 1 female; Hajnáčka (Ajnácskő): Sás Bikk, 21. 04. 2019, 1 female; Čamovce (Csomatelke): Vidosza, 25. 04. 2019, 1 female. Frequent. Larva on Rosa spp.

Sterictiphora longicornis Chevin, 1982: Jestice (Jeszte): Nagy-Somos, 03. 05. 2019, 1 female. Sporadic. Hostplants unknown, Adults associated with Prunus spinosa.

\section{Tenthredinidae}

\section{Dolerinae}

Dolerus (Dicrodolerus) vestigialis (Klug, 1818): Petrovce (Gömörpéterfala): Malaise trap, 30. 04. - 30. 06. 2019, 2 males; Hostice (Gesztete), valley west of the village, 03. 05. 2019, 1 female; Čamovce (Csomatelke): Szentfali, 24. 04. 2019, 2 males; Jestice 
(Jeszte), 03. 05. 2019, 3 females. Common. Hostplants: Equisetum palustre, E. sylvaticum, E. arvense and E. pratense.

Dolerus (Oncodolerus) eversmanni W. F. Kirby, 1882: Čamovce (Csomatelke): Rákos, 07. 06. 2019, 1 female; Čamovce (Csomatelke): Szentfali, 24. 04. 2019, 1 male. Frequent. Larva on Equisetum arvense and E. palustre.

Dolerus (Dolerus) aericeps Thomson, 1871: Čamovce (Csomatelke): Rákos, 07. 06. 2019, 1 female. Frequent. Larva on Equisetum palustre.

Dolerus (Dolerus) germanicus germanicus (Fabricius, 1775): Čamovce (Csomatelke): Rákos, 07. 06. 2019, 1 female. Common. Larva on Equisetum arvense and E. palustre.

Dolerus (Poodolerus) brevicornis Zaddach, 1859: Čamovce (Csomatelke): Istenhegye, 20. 04. 2019, 1 female. Sporadic. Hostplant: Carex spp.

Dolerus (Poodolerus) gonager (Fabricius, 1781): Šurice (Söreg): Bagoly-vár, 21. 04. 2019, 1 female. Common. Larva on Graminae.

Dolerus (Poodolerus) nigratus (O.F. Müller, 1776): Čamovce (Csomatelke): László hegy, 18. 04. 2019, 1 female, 1 male.; Tachty (Tajti) valley of Meleg-hill, 01. 05. 2019, 1 female. Common. Larva on Gramineae including cereals.

Dolerus (Poodolerus) picipes (Klug, 1818): Hajnáčka (Ajnácskő): Sás Bikk 21. 04. 2019, 1 female; Tachty (Tajti) valley of Gorva brook, 03. 05. 2019, 1 female. Frequent. Larva on Graminae.

Dolerus (Poodolerus) puncticollis Thomson, 1871: Čamovce (Csomatelke): Lászlóhegy, 18. 04. 2019, 1 female; Hajnáčka (Ajnácskő): Sás Bikk 21. 04. 2019, 1 female. Common. Larva on Graminae including cereals.

\section{Selandrinae}

Nesoselandria morio (Fabricius, 1781): Jestice (Jeszte): Nagy-Somos, 03. 05. 2019, 1 male; Jestice (Jeszte): Nagy-Somos 03. 05. 2019, 1 female. Frequent. Hostplants: Brachytecium reflexum, Ceratodon purpureus, Chenopodium album, Dicranum scoparium, Fragaria vesca, Hedwigia ciliata, Myosotis arvensis, Plagiomnium cuspidatum, Plagiothecium denticulatum, Polygonum aviculare, Polytrichum commune, Pseudobryum cinclidiodes, Sanionia uncinata, Stellaria media, Veronica chamaedrys and V. officinalis.

\section{Allantinae}

Allantus (Emphytus) didymus (Klug, 1818): Gemerské Dechtáre (Détér), 02. 05. 2019, 1 female; Jestice (Jeszte), Nagy-Somos, 03. 05. 2019, 1 male. Sporadic. Larva on Sanguisorba minor: old records from Rubus and Rosa spp. need checking. (LisTON, 2004).

Allantus (Emphytus) cingulatus (Scopoli, 1763): Hajnáčka (Ajnácskő): Sás Bikk 26. 05. 2019, 1 female. Frequent. Larva on Fragaria and Rosa spp.

Allantus (Emphytus) cinctus (Linné, 1758): Petrovce (Gömörpéterfala): Fenek, 30. 04. 2019, 1 male; Camovce (Csomatelke): Szentfali, 24. 04. 2019, 1 male. Frequent. Hostplants: Rosa spp.

Ametastegia (Ametastegia) equiseti (Fallén, 1808): Petrovce (Gömörpéterfala): Malaise trap, 30. 04. - 30. 06. 2019, 3 males. Frequent. Larva on Chenopodium album, Lythrum salicaria, Polygonum persicaria and Rumex acetosella.

Ametastegia (Ametastegia) glabrata (Fallén, 1808): Petrovce (Gömörpéterfala): Papberki, 25. 04. 2019, 1 male. Frequent. Larva on Chenopodiaceae, Polygonaceae, Plantago, Salix, Lithrum, Ribes and Rubus spp.

Athalia bicolor Serville, 1823: Hajnáčka (Ajnácskő), Tilic Hill, 20. 05.2018, 1 female. Frequent. Hostplant: Ranunculus spp. 
Athalia circularis (Klug, 1815): Petrovce (Gömörpéterfala): Malaise trap, 30. 04. - 30. 06. 2019, 3 females. Frequent. Hostplants: Arctium lappa, Ajuga reptans, Veronica beccabunga, $V$. longifolia, $V$. officinalis, Alliaria petiolata, Glechoma hederacea, Melampyrum, Capsella and Lycopus spp.

Athalia cordata Serville, 1823: Petrovce (Gömörpéterfala): Malaise trap, 30. 04. - 30. 06. 2019, 1 male; Tachty (Tajti) valley of Gortva stream, 01. 05. 2019, 1 female. Common. Larva on Misopates orontinum, Antirrhinum majus, Ajuga reptans, Teucrium scorodonia and Plantago spp.

Empria liturata (Gmelin, 1790): Petrovce (Gömörpéterfala): Malaise trap, 30. 04. 30. 06. 2019, 1 male; Jestice (Jeszte): Nagy-Somos 03. 05. 2019, 1 female; Petrovce (Gömörpéterfala): Fenek, 30. 04. 2019, 3 females, 1 male; Čamovce (Csomatelke): László hegy, 18. 04. 2019, 2 females; Čamovce (Csomatelke): Szentfali, 24. 04. 2019, 1 female; Tachty (Tajti) valley of Meleg-hill, 01. 05. 2019, 1 female. Frequent. Hostplants: Fragaria and Geum spp.

Empria sexpunctata (Serville, 1823): Petrovce (Gömörpéterfala): Fenek, 30. 04. 2019, 1 female, 1 male; Čamovce (Csomatelke): László hegy, 18. 04. 2019, 1 female. Frequent. Larva on Geum spp.

Taxonus agrorum (Fallén, 1808): Petrovce (Gömörpéterfala): Fenek, 30. 04. 2019, 1 female. Frequent. Hostplants: Rubus idaeus and R. caesius.

\section{Heterarthrinae}

Fenusella glaucopis (Konow, 1907): Čamovce (Csomatelke): Rákos, 20. 04. 2019, 1 female. Rare. Hostplants: Populus alba, P. tremula, P. nigra.

\section{Blennocampinae}

Blennocampa phyllocolpa Viitasaari \& Vikberg, 1985: Petrovce (Gömörpéterfala): Fenek, 30. 04. 2019, 1 female. Frequent. Larva rolls the leaves of Rosa spp.

Eutomostethus ephippium (Panzer, 1798): Petrovce (Gömörpéterfala): Malaise trap, 30. 04. - 30. 06. 2019, 5 males; Jestice (Jeszte), Nagy-Somos, 03. 05. 2019, 2 males, 1 female Tachty (Tajti) valley of Gortva stream, 01. 05. 2019; 2 males; Jestice (Jeszte), 03. 05. 2019, 2 females. Common, larva on Poaceae.

Halidamia affinis (Fallén, 1807): Čamovce (Csomatelke): Szentfali, 24. 04. 2019, 1 female. Frequent. Hostplants: Galium aparine and G. molugo.

Stethomostus fuliginosus (Schrank, 1781): Petrovce (Gömörpéterfala): Malaise trap, 30. 04. - 30. 06. 2019, 4 females.; Petrovce Gömörpéterfala): near Almáspuszta, 30. 04. 2019, 1 female. Sporadic. Larva on Helleborus spp., Ranunculus acris.

Monophadnus pallescens (Gmelin, 1790): Tachty (Tajti) valley of Gortva stream, 01. 05. 2019, 1 female; Čamovce (Csomatelke): Rákos, 20. 04. 2019, 1 female. Common. Hostplants: Ranunculus acris, R. repens, R. lanuginosus and Anemone nemorosa.

Pareophora pruni (Linné, 1758): Čamovce (Csomatelke): Rákos, 20. 04. 2019, 1 female. Frequent. Larva on Prunus spinosa.

Stethomostus fuliginosus (Schrank, 1781) Jestice (Jeszte): Nagy-Somos 03. 05. 2019, 1 female. Frequent. Larva on Ranunculus acris, R. repens and R. sceleratus.

\section{Tenthredininae}

Aglaostigma (Astochus) aucupariae (Klug, 1817): Čamovce (Csomatelke): László hegy, 18. 04. 2019, 1 female, 1 male; Šurice (Sőreg): Bagoly-vár, 21. 04. 2019, 1 male. Common. Larva on Galium mollugo and G. boreale.

Aglaostigma (Astochus) fulvipes (Scopoli, 1763): Petrovce (Gömörpéterfala): Fenek, 30.04. 2019, 1 female. Common. Larva on Galium mollugo and G. verum.

Macrophya (Macrophya) albicincta (Schrank, 1776): Jestice (Jeszte), 03. 05. 2019, 1 
female; Stará Bašta (Óbást), Nagy-mocsaras, 09. 06. 2019, 1 female; Čamovce (Csomatelke): Rákos, 07. 06. 2019, 1 female; Hajnáčka (Ajnácskő): Sás Bikk, 26. 05. 2019, 1 female, Petrovce (Gömörpéterfala): Fenek, 30. 04. 2019, 4 females; Jestice (Jeszte), Nagy-Somos 03. 05. 2019, 5 females, 1 male. Common. Hostplants: Sambucus ebulus, S. nigra, S. racemosa, Valeriana officinalis and Viburnum opalus.

Macrophya (Macrophya) duodecimpunctata duodecimpunctata (Linné, 1758): Čamovce (Csomatelke): Rákos, 07. 06. 2019, 5 females; Petrovce (Gömörpéterfala): Fenek, 30. 04. 2019, 1 male. Common. Hostplants: Graminae, Cyperaceae and Carex spp.

Macrophya (Macrophya) montana (Scopoli, 1763): Hajnáčka (Ajnácskő): Sás Bikk 26. 05. 2019, 4 females (Fig. 5). Common. Hostplant: Rubus caesius.

Macrophya (Macrophya) postica (Brullé, 1832): Šurice (Söreg): below the Pogány castle, 21. 05. 2018, 1 male. Frequent. Hostplant unknown.

Macrophya (Pseudomacrophya) punctumalbum (Linné, 1767): Čamovce (Csomatelke): Bodoszó, 24. 05. 2019, 1 female. Sporadic. Hostplants: Fraxinus spp. and Ligustrum spp. Pachyprotasis rapae (Linné, 1767) Čamovce (Csomatelke): Szentfali, 24. 04. 2019, 1 female. Regularly common, frequently dominant species however in 2019, only one specimen was collected. Hostplants: Solanum tuberosum, Pedicularis palustris, Angelica sylvestris, Veronica beccabunga, Betonica officinalis, Corylus avellana, Salix caprea, Fraxinus excelsior, Tussilago farfara, Symphoricarpos albus, Scrophularia, Solidago, Verbascum, Origanum, Atropa, Sarothamnus, Senecio, Polygonum, Aspidium, Epilobium, Hypericum, Galeopsis, Mentha, Polystichum, Plantago, Quercus and Stachys spp.

Rhogogaster (Rhogogaster) scalaris (Klug, 1817): Jestice (Jeszte): Nagy-Somos 03. 05. 2019, 2 females; Camovce (Csomatelke): Szentfali, 24. 04. 2019, 1 male.; Surice (Söreg): Bagoly-vár, 21. 04. 2019, 1 male. Frequent. Hostplants: Pteridium aquilinum, Alnus glutinosa, Circaea, Prunus spp., Ranunculus spp., Rosa spp., Salix alba, S. purpurea, Stellaria spp., Filipendula ulmaria, Populus tremula, Padus spp., Betula spp., Corylus avellana and Sorbus spp.

Rhogogaster (Cytisogaster) picta (Klug, 1817): Gemerské Dechtáre (Détér): NagymálBérc 02. 05. 2019, 1 female; Tachty (Tajti) valley of Meleg-hill, 01. 05. 2019, 1 female. Sporadic. Cytisus scoparius, C. nigricans, Genista germanica and G. tinctoria.

Siobla sturmii (Klug, 1817): Tachty (Tajti) valley of Meleg-hill, 23. 05. 2019, 2 males. Larva on Impatiens nolitangere. Sporadic.

Sciapteryx consobrina (Klug, 1816): Čamovce (Csomatelke): László hegy, 18. 04. 2019, 1 female. Generally frequent, locally common. Larval hosts: Adoxa spp., Anemone spp. and Ranunculus ficaria.

Tenthredo (Temuledo) temula Scopoli, 1763: Hajnáčka (Ajnácskő): Sás Bikk 26. 05. 2019, 1 male. Frequent, locally common. Larva on Ligustrum and Origanum spp.

Tenthredo (Eurogaster) mesomela Linné, 1758: Stará Bašta (Óbást): Nagy-mocsaras, 09. 06. 2019, 1 femaleç Čamovce (Csomatelke): Bodoszó, 22. 05. 2019, 1 female. Frequent. Larval hosts: Polygonum persicaria, Arctium lappa, Heracleum spp., Ranunculus spp., Epilobium spp., Rumex spp, Salix spp., Veronica sp., Tussilago spp., Petasites sp., Senecio sp., Solidago sp., and Stachys spp.

Tenthredo (Zonuledo) zonula Klug, 1817: Hajnáčka (Ajnácskő), 26. 05. 2019, 4 females. 1 male; Čamovce (Csomatelke): Bodoszó, 22. 05. 2019, 1 female; Tachty (Tajti) valley of Meleg-hill, 01. 05. 2019, 1 female; Jestice (Jeszte): Várhegy, 01. 05. 2019, 1 male. Frequent. Hostplant: Hypericum perforatum.

Tenthredopsis nassata (Linné, 1767): Petrovce (Gömörpéterfala): Malaise trap, 30. 04. - 30. 06. 2019, 1 female; Gemerské Dechtáre (Détér): Nagymál-Bérc, 24. 05. 2019, 1 female. Frequent. Hostplants: Dactylis glomerata, Deschampsia caespitosa, D. calmagrostis, Flexuosa spp., Holcus spp., Lolium perenne, Agropyron spp., Carex spp., 


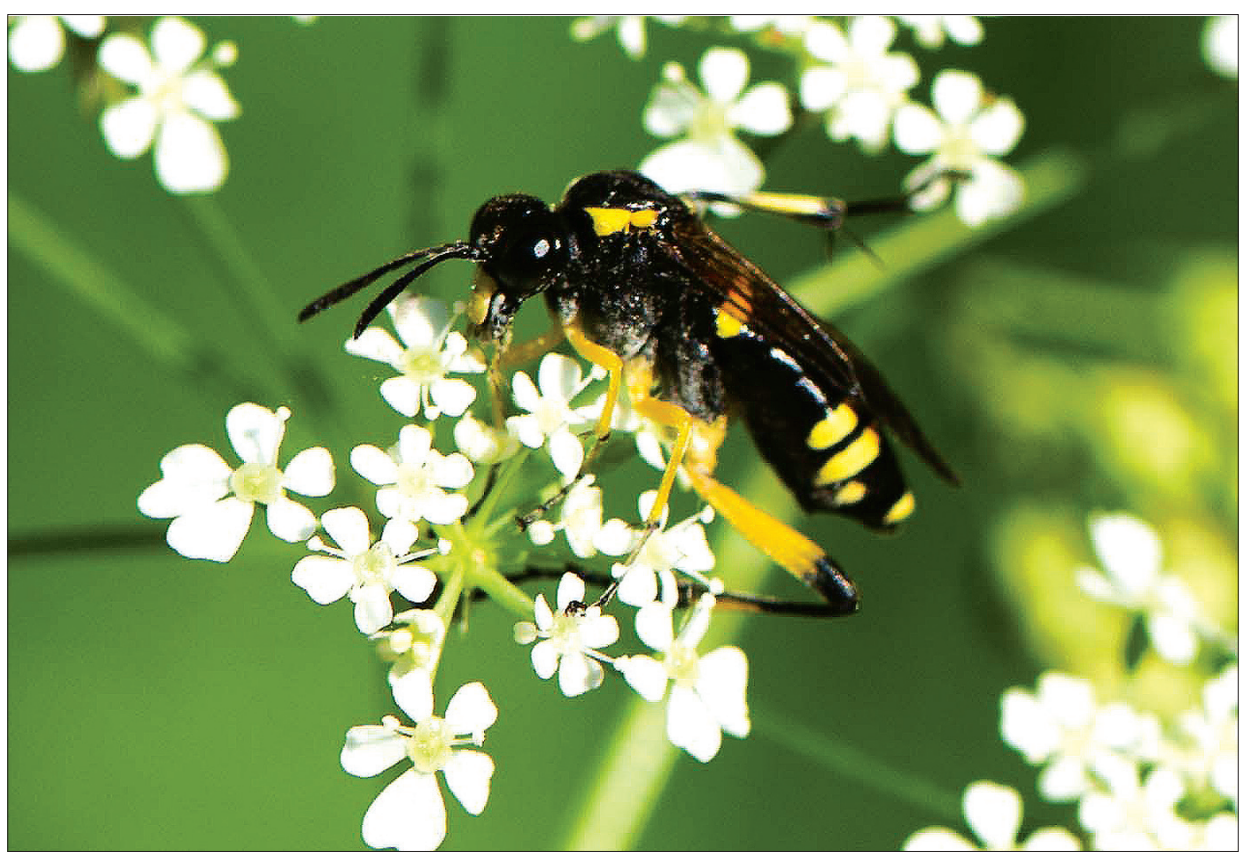

Fig. 5: Macrophya montana (Scopoli, 1763) at Hajnáčka (Ajnácskő)

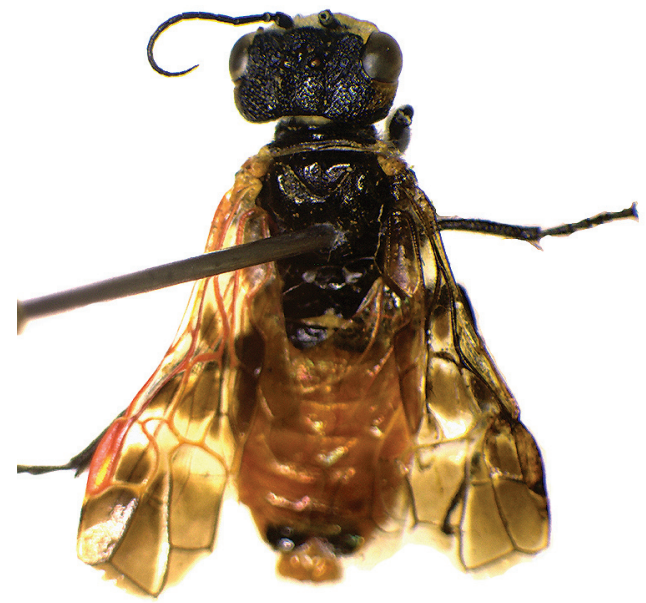

Fig. 6: Pseudocephaleia praeteritorum (Semenov, 1934) in dorsal view 


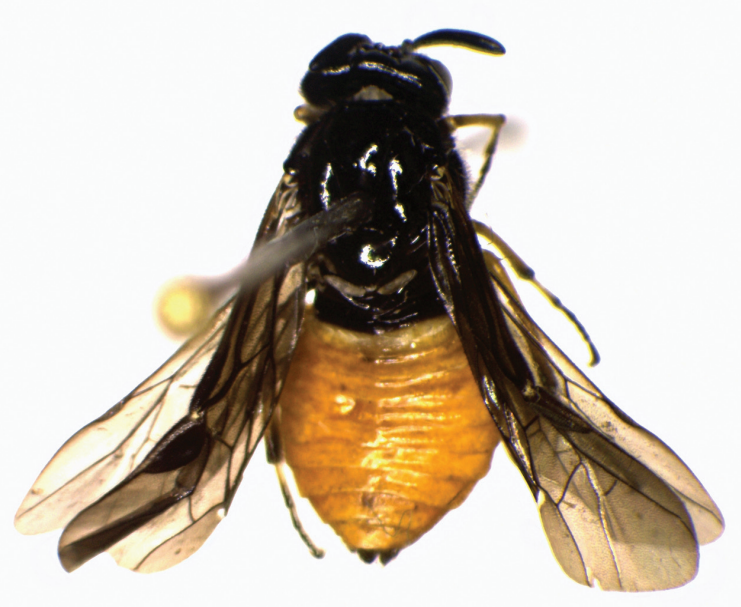

Fig. 7: Aprosthema austriacum (Konow, 1892) in dorsal view

Anthriscus silvestris and Artemisia spp.

Tenthredopsis stigma (Fabricius, 1798): Petrovce (Gömörpéterfala): Malaise trap, 30. 04. - 30. 06. 2019, 1 female; Gemerské Dechtáre (Détér): Nagymál-Bérc, 04. 05. 2019, 3 males; Gemerské Dechtáre (Détér): Nagymál-Bérc, 24. 05. 2019, 1 male; Čamovce (Csomatelke): Bodoszó, 24. 05. 2019, 1 female. Frequent. Hostplant: Triticum intermedium.

Tenthredopsis tessellata (Klug, 1817): Hajnáčka (Ajnácskő): Sás Bikk 01. 05. 2019, 1 female; Petrovce (Gömörpéterfala): Fenek, 30. 04. 2019, 1 female. Sporadic. Larva on Deschampsia, Dactylis, Aira and Lolium spp.

\section{Nematinae}

Cladius (Priophorus) brullei (Dahlbom, 1835): Čamovce (Csomatelke): László hegy, 24. 04. 2019, 1 female. Frequent. Larva on Rubus spp.

Cladius (Priophorus) compressicornis (Fabricius, 1804) (Known as Priophorus pallipes Serville, 1823): Čamovce (Csomatelke): László hegy, 18. 04. 2019, 1 female. Frequent, insect pest. Hostplants: Betula, Cotoneaster, Prunus, Rubus, Sorbus, Fragaria, Crataegus, Corylus and Rosa spp.

Cladius (Cladius) pectinicornis (Geoffroy, 1785): Tachty (Tajti) valley of Meleg-hill, 01. 05. 2019, 1 female, 1 male. Frequent. Hostplant: Rubus spp.

Euura calcicola (Benson, 1948): Tachty (Tajti) valley of Meleg-hill, 01. 05. 2019, 1 female. Rare. Hostplant unknown. New record for Slovakia.

Euura leucosticta (Hartig, 1837): Petrovce (Gömörpéterfala): Malaise trap, 30. 04. 30. 06. 2019, 6 males. Frequent. Salix aurita, S. caprea, S. atrocinerea and S. cinerea.

Euura myosotidis (Fabricius, 1804): Petrovce (Gömörpéterfala): Malaise trap, 30. 04. - 30. 06. 2019, 1 female, 2 males; Jestice (Jeszte), 03. 05. 2019, 1 female; Petrovce (Gömörpéterfala): Fenek, 06. 07. 2019, 1 female; Čamovce (Csomatelke), 20. 06. 2019, 1 female. Common. Larval hosts: Onobrychis and Trifolium spp.

Euura obducta (Hartig, 1837): Petrovce (Gömörpéterfala): Malaise trap, 30. 04. - 30. 
06. 2019, 1 female. Sporadic. Larva on Gramineae (Poa, Festuca spp.) and Carex.

Euura pavida (Serville, 1823): Tachty (Tajti): valley of Gortva stream, 01. 05. 2019, 1 female.

Euura tibialis (Newman, 1837): Chrámec (Harmac): Farkas völgy, 06. 07. 2019, 2 females. Frequent. Larva on Robinia pseudacacia.

Euura fallax (Serville, 1823) known as Pachynematus xanthocarpus (Hartig, 1840): Hajnáčka (Ajnácskő), 30. 04. 2019, 1 male; Jestice (Jeszte): Nagy-Somos, 03. 05. 2019, 1 female. Frequent. Larva on Graminae.

Hoplocampa crataegi (Klug, 1816): Tachty (Tajti): valley of Gortva stream, 01. 05. 2019, 17 females, 1 male. Frequent. Hostplants: Crataegus spp.

Hoplocampa minuta (Christ, 1791): Tachty (Tajti): valley of Gortva stream, 01. 05. 2019, 1 female. Frequent. Larva on Prunus domestica, P. armeniaca, P. instita, P. avium and P. spinosa.

Pristiphora (Pristiphora) pallidiventris (Fallén, 1808): Tachty (Tajti) valley of Meleghill, 01. 05. 2019, 1 female; Jestice (Jeszte): Várhegy, 01. 05. 2019, 1 female. Frequent. Larva on Geum, Potetilla, Rubus and Filipendula spp.

Pristiphora (Pristiphora) armata (C.G. Thomson, 1862): Tachty (Tajti) valley of Meleg-hill, 01. 05. 2019, 2 females; Tachty (Tajti) valley of Gorva brook, 12. 06. 2019, 1 male. Frequent. Larva on Crataegus spp.

Pristiphora (Pristiphora) subbifida (C. G. Thomson, 1871): Čamovce (Csomatelke): Szentfali, 24. 04. 2019, 2 females. Sporadic. Hostplants: Acer campestre, sometimes $A$. pseudoplatanus and A. orientale.

\section{Discussion}

\section{Dominant species}

Dominant species are Xyela (Xyela) graeca J.P.E.F. Stein, 1876 with 11 collected specimens, Dolerus (Dicrodolerus) vestigialis (Klug, 1818) with 9 specimens, Empria liturata (Gmelin, 1790) with 9 specimens, Eutomostethus ephippium (Panzer, 1798) with 12 specimens, Hoplocampa crataegi (Klug, 1816) with 18 specimens and Macrophya (Macrophya) albicincta (Schrank, 1776) with 10 specimens. These species make $36 \%$ of the total material

\section{Rare species and new records}

Pseudocephaleia praeteritorum (Semenov, 1934) (Fig. 6) First record for Slovakia. Palaearctic species. The species is known from Albania, Azerbaijan, China, Croatia, Italy, Romania and Turkey. Only 2 specimens are known from the Carpathian Basin so far, one female from Vászoly (North of Lake Balaton) collected on 23rd April 1984 and one female from Borosjenő (Ineu, West Transylvania) collected on 10th April 1921.

Euura calcicola (Benson, 1948) First record for Slovakia and even for the Carpathian Basin. West Palaearctic species, known from Austria, Czech Republic, France, Germany, Great Britain, Ireland, Sweden and Switzerland. The three recorded males, published by ZOMBORI (2016) from Hungary, after genitalia studies, proved to be identical with Euura clitellata (Serville, 1823), better known as Pachynematus clitellatus (Serville, 1823).

Aprosthema austriacum (Konow, 1892) (Fig. 7) First record for Slovakia. West Palaearctic species, known from Austria, Croatia, Finland, Germany, Greece, Hungary, Italy, Romania, Spain, Sweden and Ukraine. In Hungary, this species sporadically occur, 
rather rare, known from Budapest: János-hegy and Hársbokorhegy, Mecsek, Simontornya, Szilvásvárad and Hetes. From the Romanian Carpathian Mountains known from Lepsa and we have indefinite records from Burgenland (Austria) and Subcarpathia (Ukraine) either.

Fenusella glaucopis (Konow, 1907) Rare species. From Slovakia known only from PR Ostrov Kopáč (Kopács sziget). Also known from Trepcza k. Sanoka from South Poland and we have indefinite record from Croatia.

\section{References}

ACHTERBerG, C. 2013: Hymenoptera in Fauna Europaea version 2.6.2. http://www.faunaeur.org. last accessed 5th August 2019.

Achterberg, C. van \& B. van Aartsen 1986: The European Pamphiliidae (Hymenoptera: Symphyta), with special reference to The Netherlands. - Zoologische Verhandelingen Leiden 234: 1-98.

Biely, A., Bezák ,V., Elečko, M., Gross, P., Kaličiak, M., KonečnÝ, V., Lexa, J., Mello, J., NemČok, J., Polák, M., Potfaj, M., Rakús, M., Vass, D., VozÁr, J., VozÁr, A. 2002 Geologická stavba 1 : 500000. Atlas krajiny Slovenskej republiky, Bratislava: Ministerstvo životného prostredia SR, 2002. ISBN8088833-27-2, s. 124-125.

Bolfík, J. et al. 1990: Gemer-Malohont I. Príroda. Martin: Osveta, pp. 760.

Csiky, J. 2004: A Karancs, a Medves-vidék és a Cerová Vrchovina (Nógrád-Gömöri Bazaltvidék) flóra- és vegetációtérképezése - Pécs. $451 \mathrm{pp}$.

Gyurkovics, H. \& Haris, A. 2012: Sawflies (Hymenoptera: Symphyta) from Szeged and its surroundings (SE Hungary) - Natura Somogyiensis 22: 163-182.

Haris, A. 2001: Revisional list of the Hungarian Nematinae with the description of three new species (Hymenoptera: Tenthredinidae). - Folia Entomologica Hungarica 62: 95-114.

Haris, A.. 2006: Study on the Palaearctic Pristiphora species (Hymenoptera: Tenthredinidae) - Natura Somogyiensis 9: 201-277.

Haris, A. 2009: Sawflies of the Zselic Hills, SW Hungary (Hymenoptera: Symphyta) - Natura Somogyiensis 15: $127-158$.

Haris, A. 2010: Sawflies of the Vértes Mountains (Hymenoptera: Symphyta) - Natura Somogyiensis 17: 209-238.

Haris, A. 2011: Sawflies of the Börzsöny Mountains (North Hungary) (Hymenoptera: Symphyta). - Natura Somogyiensis 19: 149-176.

Haris, A. 2012: Sawflies of Belső-Somogy (Hymenoptera: Symphyta) - Natura Somogyiensis 22: 141-162.

Haris, A. 2018a: Second contribution to the sawflies of Belső Somogy (Hymenoptera: Symphyta) - Natura Somogyiensis 31: 45-62.

HARIS A. 2018b: Sawflies from Külső-Somogy, South-West Hungary (Hymenoptera: Symphyta). - Natura Somogyiensis 32: 147-164.

Horváth, G.\& GaÁlová, K. 2007 Éghajlati viszonyok, In: KIs G. \& BAráz Cs. (Eds.): A Karancs-Medves és a Cseres-hegység. Bükki N. P. Eger, pp. 91-92.

Prous, M.; Blank, S.; Goulet, H.; Heibo, E.; Liston, A.; Malm, T.; Nyman, T.; Schmidt, S.; Smith, D.; Vardal, H.; Vittasaari, M.; Vikberg, V. \& Taeger, A. 2014: The genera of Nematinae (Hymenoptera, Tenthredinidae) - Journal of Hymenoptera Research 40: 1-69.

Roller, L. 1993: New records of sawflies (Hymenoptera: Symphyta) from Slovakia. - Entomological Problems 24(2): 81-84.

Roller, L., 1994: Faunistics records. Symphyta. - Entomological Problems 25(2): 24.

Roller, L. 1996: New records of sawflies (Hymenoptera, Tenthredinidae) in Slovakia. - Biologia, Bratislava 51(1): 549-550.

Roller, L. 1998: Sawfly (Hymenoptera, Symphyta) community in the Devínska Kobyla National Nature Reserve. - Biologia, Bratislava 53(2): 213-221.

Roller, L. 1999a: Spoločenstvá hrubopásych (Hymenoptera: Symphyta) vybraných zoogeografických regiónov Slovenska. PhD thesis, Ústav zoológie, Slovenská akadémia vied, Bratislava, 180 pp.

Roller, L. 1999b: First records of Nematinae (Hymenoptera, Symphyta, Tenthredinidae) in Slovakia. Biologia, Bratislava 54(5): 599-600. 
RolleR, L. 1999c: Faunistic records from Slovakia. Hymenoptera: Symphyta: Tenthredinidae: Nematinae. Entomological Problems 30(1): 30.

Roller, L. 1999d: Faunistic records from Slovakia. Hymenoptera: Symphyta: Tenthredinidae: Nematinae. Entomological Problems 30(1): 52.

Roller, L., 1999e: Check list of the sawflies (Hymenoptera: Symphyta) of Slovakia. - Entomological Problems 30(2): 37-48.

Roller, L., 2000a Zubačkovité (Megalodontesidae) - hrubopáse xerotermov. Hmyz 1(1): 17-18.

Roller, L., 2000b First records of Blasticotomidae, Tenthredinidae, Pamphiliidae (Hymenoptera) from Slovakia. - Biologia, Bratislava 55(5): 561-562.

Roller, L., 2000c Súčasný stav poznania fauny hrubopásych (Hymenoptera, Symphyta) na Slovensku. Správy Slovenskej zoologickej spoločnosti 18: 109-114.

Roller, L., 2001: Príspevok k poznaniu hrubopásych (Hymenoptera, Symphyta) a rohačkovitých (Diptera, Sciomyzidae) prírodnej pamiatky Mitická slatina. p. 32-36. In: MáJSKY, J. (ed.), Zborník výsledkov inventarizačného výskumu prírodnej pamiatky Mitická slatina. Občianske združenie Pre Prírodu, Trenčín, 99 pp.

Roller, L. 2004. Hrubopáse blanokrídlovce (Hymenoptera, Symphyta) Tematínskych kopcov. - Entomofauna Carpathica 16: 56-64.

Roller, L., 2005: Blanokrídlovce (Hymenoptera): hrubopáse (Symphyta). 117-123 In: Fauna Devínskej Kobyly. APOP, Bratislava, 181 pp.

Roller, L., 2006a: Seasonal flight activity of sawflies (Hymenoptera, Symphyta) in submontane region of the West Carpathians, Central Slovakia. - Biologia, Bratislava 61(2): 193-205.

Roller, L., 2006b: Hrubopáse blanokrídlovce (Hymenoptera, Symphyta) Tematínskych vrchov - zhrnutie faunistického výskumu. p. 53-55. In: K. RAJCOvá (ed.): Najvzácnejšie prírodné hodnoty Tematínskych vrchov. Zborník výsledkov inventarizačného výskumu územia európskeho významu Tematínske vrchy. KOZA, Trenčín a Pre Prírodu, Trenčín, 101 pp.

Roller, L. 2010. Hrubopáse blanokrídlovce (Hymenoptera: Symphyta) PR Šúr, pp. 215-235. In: Majzlan, O., Vidlička, L. (eds). Príroda rezervácie Šúr. Ústav zoológie SAV, Bratislava, 410 pp.

Roller, L., LuKáš, J., 1999: New records of sawflies (Hymenoptera, Symphyta) in Slovakia. - Biologia, Bratislava 54(2): 225-228.

Roller L., Beneš K., Blank S. M., Holuša J., Jansen E., Jänicke M., Kaluza S., Kehl A., Kehr I., Kraus M., Liston A. D., Nyman T., Nie H., Savina H., Taeger A., Wei M., 2006: Contribution to the knowledge of sawfly fauna (Hymenoptera, Symphyta) of the Low Tatras National Park in Central Slovakia. - Naturae Tutela 10: 57-72.

Roller, L. and MaceK, J. 2017: Provnález Hrubopásych Blanokrídlovcov (Hymenoptera, Symphyta) na Slovensku - Entomofauna carpathica, 29(1): 53-63.

Roller, L. \& OlšovskÝ, T. 2012: Prvonálezy hrubopásych blanokrídlovcov (Hymenoptera, Symphyta) v slatinných lesoch s tavol’níkom vŕbolistým (Spiraea salicifolia) v Borskej nížine. - Entomofauna carpathica 24(1): 15-20.

Zhelochovtsev, A. N. 1988: Otryad Hymenoptera - Pereponchatokrylye, Podotryad Symphyta Sidyachebryukhie, 7-234. In: Medvedev, K. H. (ed.) Opredelitel nasekomykh evropeiskoi chasti SSSR, Vol. 3 Hymenoptera, Part 6, Nauka, Leningrad.

Zombori, L. 2016: Levéldarázs-alkaúak IV. Tenthredinoidea IV. In Fauna Hungariae 9. Hymenoptera I. Fauna Hungariae 174. 3/c booklet. Mondat Kft. and Hungarian Natural History Museum, Budapest. 160 pp. 
